\title{
An Integrated Approach to Characterizing ZnO Nanostructures by Thin-film XRD Techniques and SEM
}

\author{
Nandita Nag and Jingyue (Jimmy) Liu
}

Center for Nanoscience and Department of Physics \& Astronomy, University of Missouri-St. Louis, One University Boulevard, St. Louis, Missouri 63121, USA (liuj@,umsl.edu).

Due to their versatile applications, thin films have become a significant branch of materials science and technology over the last few decades. Since structure and function of materials are related intimately, characterization of structural properties is a critical issue for thin film development. In most thin film investigations, a large fraction of the measured information could come from the substrate, complicating data interpretation. Grazing incidence x-ray diffraction (GIXRD) probes the sample up to a specific depth, depending on the incidence angle $[1,2]$. The small incidence angle causes the path traveled by the X-rays in a thin film to increase significantly; so information on the properties of thin films can be obtained. In this study, we use GIXRD to probe the structure of $\mathrm{ZnO}$ nanobelts grown on a flat surface. To obtain both morphological and structural information on the $\mathrm{ZnO}$ nanobelts we correlated the GIXRD data with SEM images obtained from the same sample regions. Pole figure analyses were performed to determine the texture of the synthesized material.

The $\mathrm{ZnO}$ nanostructures were prepared by a physical vapor evaporation-deposition method. An Ultima IV diffractometer (Rigaku Corp., Japan), equipped with a thin film diffraction attachment, was used to perform the GIXRD and the pole figure measurements. The GIXRD patterns were obtained by varying the X-ray incident angle to probe both surface and near-surface structures. A JEOL6320F field emission SEM was used to examine the morphology and size distributions of the $\mathrm{ZnO}$ nanostructures.

Figure 1 is a low magnification SEM image of $\mathrm{ZnO}$ nanostructures grown on a substrate, showing the presence of large $\mathrm{ZnO}$ nanobelts on top of the substrate. Detailed examination revealed that, in addition to the nanobelts, there was a thick layer of $\mathrm{ZnO}$ film below the nanobelts. GIXRD patterns (Fig. 2) with varying incident angles were obtained from the same region of the sample as shown in Fig. 1. The XRD patterns clearly show that both the thin film and the nanobelts exhibit the wurtzite structure of $\mathrm{ZnO}$. When the peak intensities were compared with the relative intensities of the reference pattern of bulk $\mathrm{ZnO}$ [3], the variations of intensity ratios provide information on the preferential alignment of the crystal growth. The GIXRD patterns show that the relative intensity ratios depend on the incidence angle $(\alpha)$ of the X-ray beam. At $\alpha=5^{\circ}$, the (002) peak has the highest intensity. With $\alpha=1^{\circ}$, the (002) peak intensity is significantly reduced and the (101) peak shows the highest intensity. This can be explained by the variation in the preferred orientation of the nanostructures. Based on the SEM image shown in fig. 1 we can interpret the GIXRD patterns as below. With large incidence angles, the diffraction patterns originated primarily from the highdensity $\mathrm{ZnO}$ film although the $\mathrm{ZnO}$ nanobelts on top of this film also contributed. With decreasing incidence angles, the low density $\mathrm{ZnO}$ nanobelts contributed more and more to the GIXRD pattern. Therefore, the GIXRD patterns shown in Fig. 2 suggest that the $\mathrm{ZnO}$ crystals in the thick $\mathrm{ZnO}$ film grew preferentially along the $\mathrm{ZnO}$ [001] direction and that the $\mathrm{ZnO}$ nanobelts in this particular sample did not grow with any preferential orientations. Further investigation on the presence and degree of texture of the nanostructures by stereographic projection of the pole densities indicates the presence of strong fiber texture. Details of the pole figure study will be discussed. 


\section{References}

[1] S. Matsuno et al., The Rigaku Journal, 17/No.2/ 2000.

[2] Mario Birkhlz, Thin film analysis by X-ray scattering, Willey-VCH Verlag GnbH\&Co. Weinheim, 2006.

[3] JCPDS card-1451, ZnO.

[4] This research was supported by the University of Missouri-St. Louis.
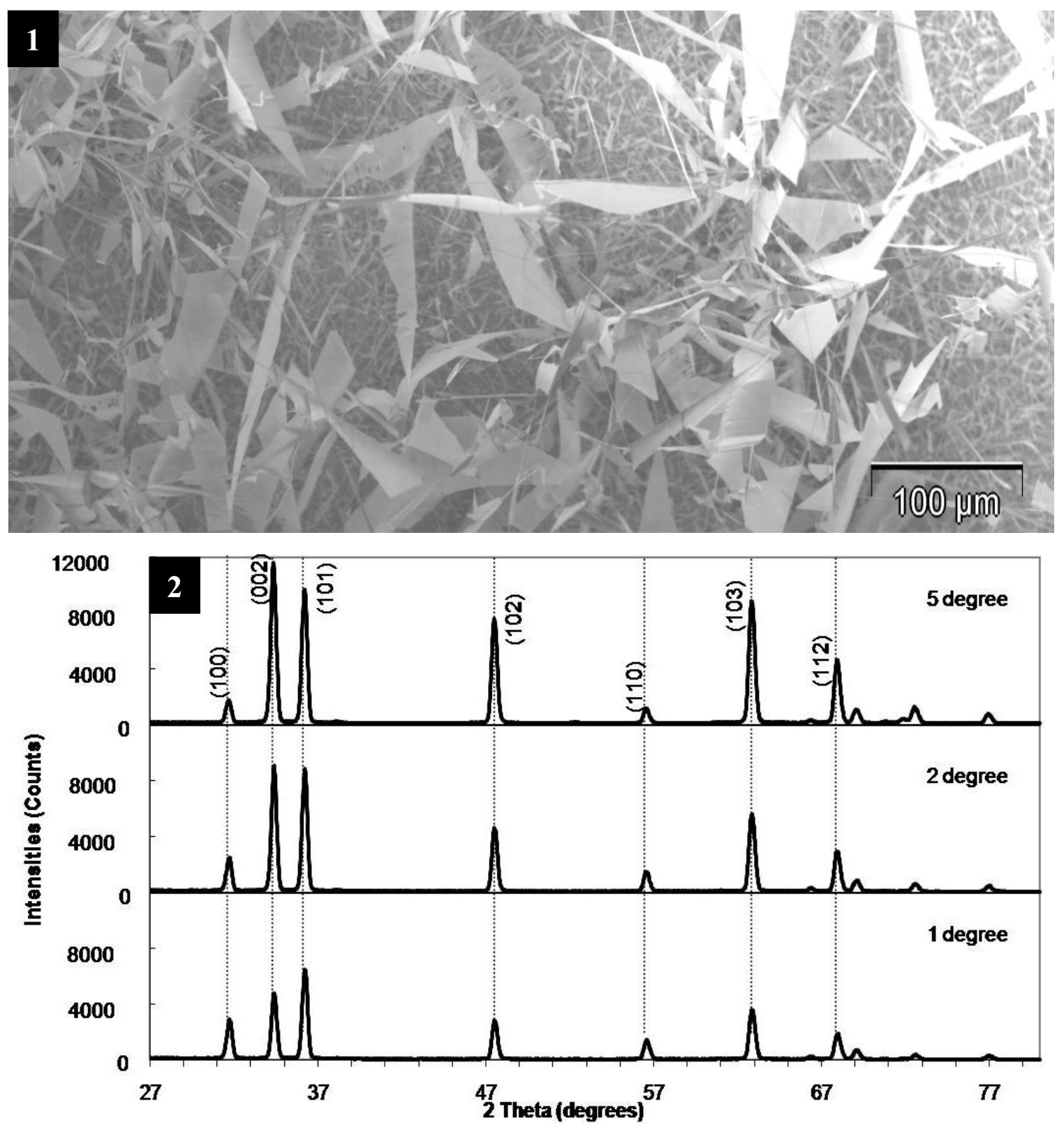

Fig.1. Low magnification SEM image shows various sizes of $\mathrm{ZnO}$ nanobelts and a thick film underneath the nanobelts.

Fig.2. Grazing incidence XRD patterns obtained from the same sample area as shown in Fig. 1, revealing the variations of intensity ratios with respect to X-ray the incidence angle. 